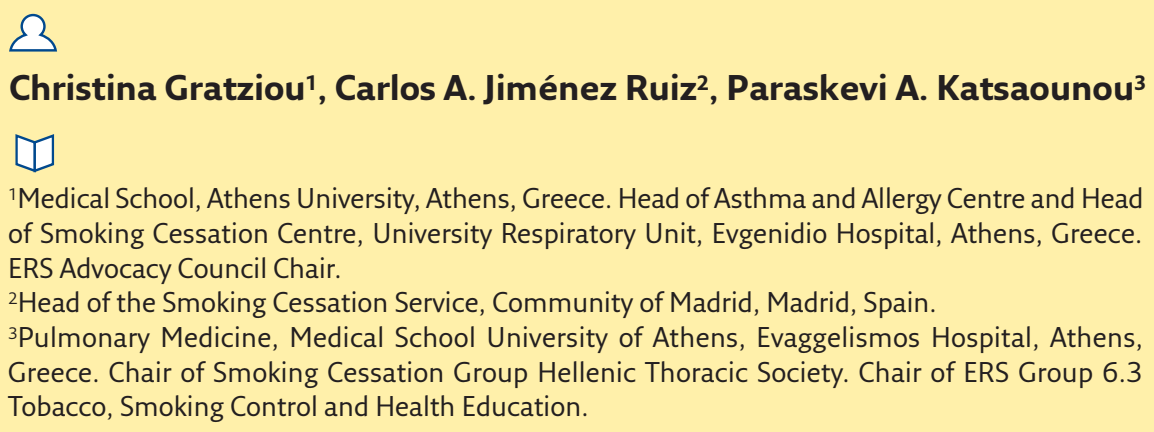

\title{
Smoking cessation using innovative techniques: course report
}

\section{Course organisers}

Christina Gratziou, Paraskevi Katsaounou, Carlos Jiménez Ruiz

Faculty: Paraskevi Argyropoulou-Pataka, Peter J. Barnes, Panagiotis Behrakis, Elif Dağlı, Bertrand Dautzenberg, Dimos Fotopoulos, Nickolaos Koulouris, loanna Mitrouska, Sofia Ravara, Sotiria Schoretsaniti, Jean-Paul Sculier, Philip Tønnesen, Serena Tonstad, Michael Toumbis, Constantine Vardavas.

\section{Introduction}

Tobacco dependence is a chronic disease of nicotine addiction, which needs to be faced and be treated properly by health professionals. Despite many years of educational efforts in many EU and non-EU countries, smoking cessation clinics are underdeveloped or do not even exist. Health professionals must view tobacco use as a medical condition and not as a habit, pleasure or lifestyle choice.

ERS has been very active in tobacco-control strategies for many years and actions on smoking cessation are considered very important according to the World Health Organization's Framework Convention on Tobacco Control, article 14.

For this reason, ERS Education organised a new 3-day educational course on smoking cessation and innovative techniques of treatment in order to offer an intensive course with specific new approaches for smoking cessation in difficult cases. The course took place on July 2-4, 2015 in Athens, Greece, with 91 participants, most of whom were clinicians. Aimed at all healthcare professionals, this course aimed to provide a comprehensive update in tobacco-related diseases and smoking cessation. The curriculum covered hot topics from smoking cessation experts to give participants new skills in the areas of motivational interviewing, smoking cessation strategies and application, the organisation and implementation of smoking cessation clinics and personalisation of treatment to match each individual's profile and needs. Also, new challenges, such as the current debates on electronic cigarettes were addressed.

The format of the course was lectures supplemented with video-recorded case presentations and interactive workshops and discussions.

A number of key opinion leaders in the field were invited as speakers and trainees, and all participants had the chance to have close discussion with them about difficult cases during the workshops.

Participants had the chance, through this course, for systematic assistance and information on standard procedures and up-to-date methods for running a smoking cessation clinic and performing a smoking cessation service. Other learning outcomes included identification of individual and social factors affecting smoking persistence, including concerns associated with special groups of smokers, such as adolescents, pregnant women and those with psychiatric and other conditions, and choosing the best pharmacological treatments for each smoker.

The need to inculcate smoking cessation in medical school curricula was also stressed as an important factor to change the attitude in the face of what could be described as a smoking epidemic seen even in medical doctors.

Motivational interviewing, needed to bring about cessation in smokers, is also a field that needs to be raised among doctors in training. Finally, the need to learn about advocacy and regulations was addressed, so as to incorporate ERS tobacco policies in participants' professional settings and contribute to their national tobacco control planning.

The interactive sessions were instrumental in better explaining specific challenges faced in real-life smoking-cessation clinics giving the
Katsaounou, Christina Gratziou, Carlos A. Jiménez Ruiz. Smoking cessation using innovative techniques: course report. Breathe 2015: 11: 255-256.

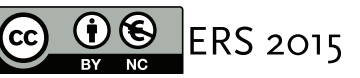

HERMES syllabus link: 


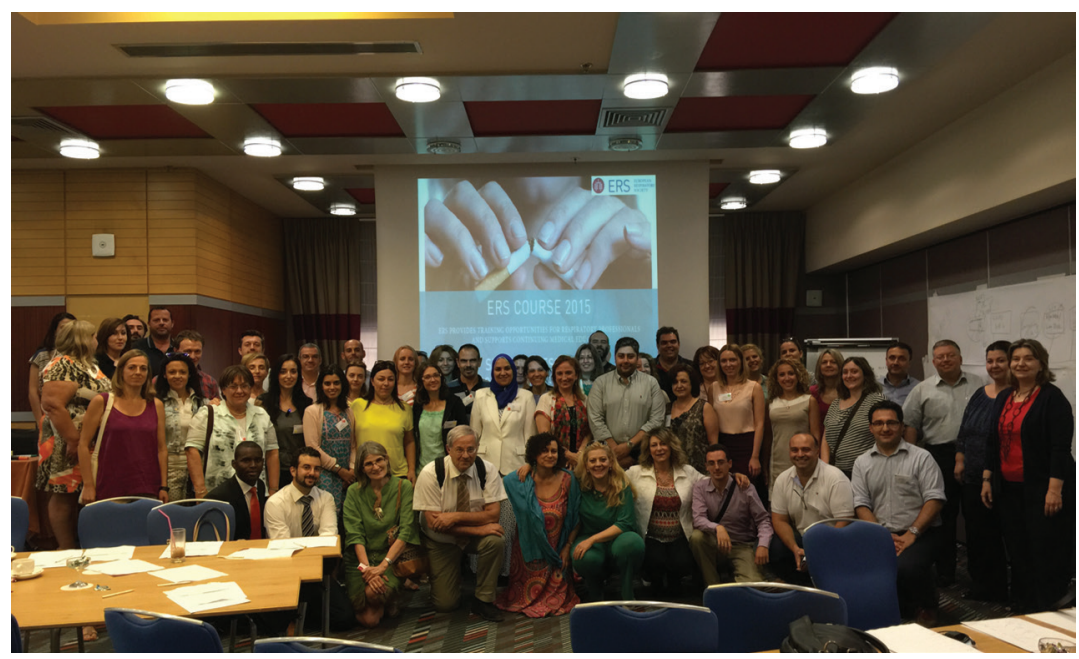

faculty the chance to clarify key areas raised from the electronic voting system and heated discussions during the various workshops. With participants from not only Europe but also Egypt, Israel, Australia and the USA, these interactive sessions gave opportunities for sharing experience and expertise with international colleagues and gave the course an international flavour.

One discussion of note surrounded the use of electronic cigarettes in smoking cessation. The controversy and the debate surrounding electronic cigarette use as a smoking cessation tool is still not completely resolved; there are some proponents that believe that the electronic cigarette use and "vaping" should not be seen as smoking, and many scientific committees support the need for more science to prove the efficacy and the safety of these products for smoking cessation.

In conclusion, the course was a great success and ERS will continue providing education on smoking cessation and will investigate new formats to have a wider and more effective impact. Role play was particularly interesting for participants and there was a general consensus that the sessions were very effective in meeting specific course needs. Future considerations include the need for more time to allow role playing in specific populations and a focus on teaching the skills involved in motivational interviewing (both the theory and practice). A number of educational workshops (EW19, 21, 23) at the ERS International Congress dealt with this latter topic.

\section{Eman Sobh, Egypt, a participant:}

This course aimed at expanding the knowledge of smoking cessation of the participants, and offered the opportunity to discuss the practical issues in fighting smoking and dealing with smokers.

The participants gained knowledge about different aspects of smoking cessation, pharmacotherapy and management of smoking cessation in special groups.

Discussions were valuable and different points were important like interviews with smokers and how to moderate interviews with motivated and nonmotivated smokers. Also, discussing different issues relating to nonpharmacological therapy.

Discussions emphasised on the central problem of how to reach persons willing or not to stop smoking and help them to stop smoking through different approaches counselling, medicine or both.

The most important point of debate in discussion was the use of electronic cigarette in smoking cessation. Some were supporting for this assuming electronic cigarette as a tool for smoking cessation and others were not, as they thought it is harmful even if less than usual cigarette and not approved, long-term effects are not known and there are no sufficient data on its harmful effects. This may be due to the lack of knowledge regarding electronic cigarette ingredients, use and longterm side effects; and most respiratory societies wouldn't support the use of electronic cigarettes in smoking cessation until sufficient data are available. There was concern that conflict of interest may participate to lack of evidence regarding electronic cigarette.

The organisers and the faculty moderated discussions in an excellent way and motivated all participants to take part in discussions.

One of the most important points raised during this course was patient integration and participation; it was practical to have an interview with true patients.

The atmosphere of the course was excellent and the faculty helped everyone to participate in discussions and active workshops. It was a good medium for communication between colleagues from different countries and to help build good relationships and research cooperation.

ERS could arrange another course in more detail discussing from a scientific point of view further issues regarding electronic nicotine delivery devices like e-cigarettes.

Many thanks to ERS. Participation in this course motivated me to continue on the way to help smoking cessation and to talk with strategy makers about improving smoking cessation clinics and availability of pharmacotherapy. The course also provided me with more knowledge about smoking cessation in special patients.

\section{Conflict of interest}

None declared. 